\title{
América Latina, las medidas de confianza mutua y de seguridad regional. Evaluación y perspectivas '
}

\section{Francisco Rojas Aravena}

El contexto internacional de posguerra fría y de globalización enfatiza en la necesidad de desarrollar elementos de carácter cooperativo como instrimento esencial, casi único para los paises medianos y pequeños del sistema intemacional para resolver los desafios planteados por la nueva agenda internacional. Aún para las superpotencias el clesarrollo de políticas asociativas aparece como un instrumento esencial al abordar muchos de los desafios inherentes a la agenda emergente en el sistema intemacional. Para poder avanzar de manera sustantiva en el tratamiento de la nueva agenda, chyos instrumentos están basados en la cooperación, es necesario resolver las dimensiones de amenazas afincadas en la agenda tradicional, vinculadas a percepciones de amenaza de carácter militar, en especial en el eje soberano territorial. La existencia de voluntad política por resolver los temas de la agenda histórica y el desarrollo de las medidas de confianza mutua y' de seguridad constituyen instrtmentos fundamentales, que han demostrado un alto nivel de eficiencia al ser aplicado de manera bilateral y regional en América Latina, durante la década de los años 90 s.

El desarrollo de las Medidas de Fomento de la Confianza Mutua y la Seguridad (MCM) es uno de los puntos principales de la agenda de seguridad internacional de América Latina. Ha sido un tema destacado de las reuniones Ministeriales de Defensa de las Américas, tanto en Williamsburg (1995) como en Bariloche (1996) y en Cartagena (1998). Los acuerdos alcanzados y las

\footnotetext{
${ }^{1}$ Presentación realizada ante la Comisión de Defensa Nacional de la Cámara de Diputados de Chile, como parte del Seminario organizado por esta corporaciôn acerca de la "Condición estratégica de Chile". Valparaíso, 18 de agosto de 1999.
} 
resoluciones adoptadas en la reunión de alto nivel de la Organización de Estados Americanos sobre el tema, que se realizó en Santiago de Chile, entre el 8 y 10 de noviembre de 1995 y en El Salvador en febrero de 1998, poseen una alta significación. La "Declaración de Santiago sobre medidas de fomento de la confianza y de la seguridad" marca un hito, tan significativo como el Compromiso de Santiago sobre la Promoción de la Democracia del año 1991. Ello se evidencia al constatar los párrafos correspondientes de la Declaración de la II Cumbre de las Américas celebrada en Santiago en abril de 1998. La reciente Asamblea General de la Organización de Estados Americanos, celebrada en el mes de junio de 1999 en Ciudad de Guatemala, ratificó el compromiso regional y hemisférico en estas materias.

Las MCM son un tema central de la diplomacia preventiva. ${ }^{2}$ Su desarrollo posibilitará establecer un proceso acumulativo de transparencia que se traducirá en la generación de Acuerdos de Seguridad con énfasis en los aspectos cooperativos en la región y el hemisferio. Su aplicación efectiva dará lugar al desarrollo un código de conducta que materializará, por medio de acciones específicas, la búsqueda de estabilidad y paz en esta región.

Antes de abordar el tema específico de medidas de confianza mutua, quisiera destacar algunos aspectos que caracterizan al sistema internacional. En primer lugar, cito al Canciller Juan Gabriel Valdés quien destaca la necesidad de mirar las oportunidades de cooperación al abordar las diversas agendas. ${ }^{3}$

"En esta nueva y compleja realidad internacional, los intereses de los diversos Estados nacionales se han diversificado, dando lugar a agendas múltiples que requieren de mecanismos que garanticen relaciones más cooperativas y solidarias entre las naciones. No sólo se trata de evitar los conflictos y las tensiones bélicas, sino también de encarar nuevos riesgos. Con ese propósito, se busca aumentar la cooperación internacional frente a temas tan cruciales como la promoción de la democracia y de los derechos humanos, el fortalecimiento de la paz, la preservación de recursos naturales, la regulación de los espacios comunes, la liberalización del comercio mundial, la lucha contra el narcotráfico y el terrorismo internacional".

- Con la posguerra fía adquirió una gran relevancia el accionar de la Diplomacia Preventiva; este concepto ocupó un lugar central en un Programa de Paz del ex Secretario General Boutros Boutros Gahli (ONU, N.Y., 1992).

3 Juan Gabriel Valdēs, discurso en el seminario sobre "Sobre la seguridad internacional a inicios del siglo XXI", organizado por el Ministerio de Defensa de Chile. Santiago, 16 de agosto de 1999). 
Con el fin de poder definir el contexto en el cual la cooperación debe operar, me permito destacar algunios aspectos que caracterizan la visión del sistema internacional desde América Latina.

1.- Hace una década o una década y media, América Latina aprendía de Europa sobre integración, sobre seguridad regional, sobre MCM. Hoy (1999), al finalizar el siglo, podemos decir que aprendimos la lección, hicimos muy bien la tarea y tenemos experiencias importantes para compartir. ${ }^{4}$

2.- El multilateralismo global, en su expresión máxima las Naciones Unidas, está en la mayor crisis de sus 50 años de vida. Perdió el apoyo de quienes eran su principal soporte valórico, político, económico: el mundo occidental, los países democráticos occidentales. Kosovo lo evidenció dramáticamente. ${ }^{5}$

3.- Una institucionalidad bien diseñada no es suficiente. No basta con tener una arquitectura compleja y un marco conceptual bien articulado. Si la institucionalidad no se ocupa, si falta voluntad política no queda nada. Esa es mi impresión de la Organización de Cooperación y Seguridad Europea (OESE) y la crisis de los Balcanes. El mejor diseño arquitectónico e institucional y la ausencia de acción total en el momento de crisis. ${ }^{6}$

4.- La globalización impone mirar desde nuevas perspectivas las relaciones de Europa-América Latina. Ellas se deben enmarcar en un sentido más amplio: la comunidad occidental. Esto impone mirar el triángulo AtlánticoEuropa, América Latina y Estados Unidos- desde nuevas perspectivas. Tenemos un sentido y una proyección valórica que está en el origen del pensamiento occidental, pero sin concreción en una comunidad político-cultural de carácter global. ${ }^{7}$ Es esto lo que permite superar el determinismo geográfico de los intereses particulares americanos, europeos o latinoamericanos. La importancia de este "bloque" y las consecuencias se expresan en una mirada sobre cualquier indicador, por ejemplo población, producto bruto, exportaciones, inversión, estabilidad, coincidencias en política internacional, etc.

\footnotetext{
4 Jorge Dominguez (ed.) Seguridad internacional, paz y democracia en el Cono Sur. FLACSO-Chile/InterAmerican Dialogue/P\&SA. FLACSO-Chile, Santiago, 1998.

Francisco Rojas Aravena, Bernardo Arévalo, Carlos Sojo, La nueva agenda de seguridad en Centroamérica. FLACSO-Guatemala/FLACSO-Chile/P\&SA. Guatemala, 1998.

$s$ Ver Revista Política Exterior No. 69 (mayo.junio 1999) y No. 70 (julio-agosto 1999). Madrid. También ver articulos sobre este tema en Revista Estudios Internacionales, julio-diciembre. Santiago, 1998.

6 Ministerio de Defensa de España. Organización para la Seguridad y Cooperación en Europa. OSCE. Documentos. Madrid, 1995.

7 Esto nos remite a generar una mirada nueva en ausencia del conflicto bipolar-al triángulo Atlántico. A inicios de agosto, durante la visita del Canciller Valdés a Washington, la Secretaria de Estado de Estados Unidos, invitó a Chile a sumarse a una iniciativa global tendiente a constituir una Comunidad de Naciones Democráticas.
} 
5.- Constatamos la ausencia de un catalizador de asociación; no hay un elemento externo que presione a la asociación. La proyección a mediano plazo en cuestiones económicas mas bien nos separa. América Latina, en particular el Mercosur, estará en una etapa que puede ser propicia para reafirmar su propio espacio, si perfecciona sus arreglos de coordinación de políticas. La Unión Europea estará anclada en el este, empantanada en los Balcanes durante, al menos, los próximos cinco años. La política agraria común también es un peso muy grande, tal como se expresa en el veto francés al mandato negociador para la Cumbre Unión Europea-América Latina. Estados Unidos al menos durante dos o tres años no tendrá fast track, con lo cual la posibilidad de una negociación más amplia en América Latina se verá reducida. El ALCA tendrá avances, pero será un proceso lento. El demandará una mayor coordinación latinoamericana que no hemos sabido desarrollar. Asia se encuentra en un proceso de reconstrucción institucional después del terremoto financiero y las posibilidades y ampliación hacia esta región del mundo no son significativas. En América Latina el mayor peso está dado en lo subregional. El Mercosur tiene una importancia gravitante como subregión específica. Es necesario recuperar la memoria histórica de la integración con un sentido de identidad más global.

6.- El uso de la fuerza. Esta es una cuestión internacional, dejó de tener una focalización nacional. La guerra es ilegal, sin embargo, tenemos más conflictos en el planeta que en período previo. La gran mayoría de ellos son intraestatales. ${ }^{8}$ Esto cambia las bases de operación de los mecanismos de prevención y afecta al concepto central del multilateralismo: la soberanía. ${ }^{9}$

Lo anterior significa reconocer una falla básica en el sistema internacional: el sistema de sanciones. Si no tenemos un sistema de sanciones adecuado, sólo queda el uso de la fuerza o bien la impunidad y la permisividad. Si prima la fuerza las asimetrías de poder son el elemento crucial. Todo sistema de alerta temprana posee un componente vinculado a las sanciones, si ellas no producen resultados efectivos falla el conjunto. ${ }^{10}$

\footnotetext{
${ }^{8}$ Chester A. Crocker, Fen O. Hampson with Pamela Aall (eds.), Managing Global Chaos. Source of and Responses to International Conflict. USP Press. Washington, D.C., 1996.

9 Aspen Institute, Managing Conflict in the Post-Cold War World: The Role of Intervention. Aspen Institute Conference on International Peace and Security. Washington, D.C., 1996.

${ }^{10}$ Alexander L. George \& William E. Simons, The Limits of Coercive Diplomacy. Westview Press. Boulder, Colorado, 1994.
} 
El tema central de la comunidad internacional y la construcción del multilateralismo futuro estará en reflexionar sobre ambas cuestiones. Esto requiere de más consenso político para su implementación y la construcción institucional.

7.- La participación parlamentaria debe ser efectiva. En el marco de la globalización se requiere la participación de nuevos actores en la definición del sistema internacional. Más allá del debate sobre la participación de la sociedad civil en este nuevo contexto, debe destacarse la necesidad de participación de los parlamentarios de manera más efectiva en este proceso. Si bien la primacía la continúan teniendo los gobiernos, la representación parlamentaria debe involucrarse en el proceso y de esta manera se asegurará la ratificación de los tratados internacionales.

Una cuestión sustantiva del nuevo sistema internacional está radicada en la definición de los nuevos bienes públicos internacionales que deben regir las relaciones globales y regionales. " Para asegurar su eficiencia es necesario que los parlamentarios participen de manera efectiva a lo largo del proceso. Ello demanda el desarrollo de políticas de Estado en las cuestiones fundamentales y el desarrollo de nuevas formas de participación de los parlamentarios en el proceso.

8.- El rol académico es fundamental. La generación de ideas, el ubicar nuevas perspectivas que sean capaces de ir más allá de la crisis del Estado y evaluar el impacto de la globalización en cada área, requiere que el mundo académico genere nuevas perspectivas, proyecciones y recomendaciones. También para el desarrollo del nuevo multilateralismo. En ese sentido, FLACSO-Chile coordina una red de investigadores en América Latina y el hemisferio. Para analizar estas temáticas y generar recomendaciones con distintas instituciones, en base a reunir a académicos, diplomáticos, militares y periodistas en una misma mesa con el único interés de pensar en un mundo mejor, con relaciones desmilitarizadas y con relaciones de cooperación como cuestiones sustantivas.

\footnotetext{
"Inge Kaul, Isabelle Grunberg \& Marc A. Stern (eds.), Global Public Goods. International Cooperation in the 215t Century. Oxford University Press/UNDP. New York, 1999.
} 


\section{Construir la confianza mutua ${ }^{12}$}

La confianza se encuentra sustentada en un activo que posee un alto grado de intangibilidad. La confianza se construye en el proceso paulatino. La confianza es incremental. Sin embargo, ella se pierde bruscamente. La reversión del proceso es acelerada. Las MCM buscan establecer un patrón de relación que otorgue crédito a las declaraciones de intención y transparente las conductas. Están diseñadas para evidenciar los actos que buscan afectar la seguridad, la integridad u otro interés vital, y diferenciarlos de otras acciones. De allí el vínculo esencial entre MCM y los procesos de verificación.

Considerando la herencia colonial y las recurrentes de tensiones fronterizas lo predictible es que ellas se repitan estableciendo un patrón de relación de alta desconfianza, amenazas y recelos. Romper con ese futuro predictible y generar un nuevo patrón de relación cimentado en la asociación es lo que las MCM posibilitan como instrumento. En el debate sobre amplitud o restricción del concepto de medidas de fomento de la confianza, privilegiamos la focalización. Estimamos que el uso de MCM debe estar radicado exclusivamente en el ámbito de la defensa. La construcción de confianza en otros ámbitos es responsabilidad de la política, la diplomacia, la economía y otras relaciones. Las MCM no deben ser confundidas con otras acciones que significan acuerdos de cooperación. El concepto sobre MCM debemos reservarlo para aquellas cuestiones que dicen relación con el uso de la fuerza y las otras medidas debemos ubicarlas en el campo de la cooperación.

Las MCM buscan conferir estabilidad a las relaciones. Establecen un proceso por medio del cual se da satisfacción concreta a los compromisos que se deben materializar en el futuro. Las MCM buscan cambiar una historia de desconfianza que implica un alto grado de riesgo, por una situación, en la cual, sobre la base de acciones que evaluamos generamos respuestas que llevan a la estabilidad y luego a la distensión. Las MCM buscan romper la historia de desconfianza que caracteriza los vínculos entre los países de la región. Si nos quedamos con una mirada en el pasado no tenemos opciones para el futuro. Si sólo confiamos en las declaraciones ponemos el futuro en una acción de fe.

\footnotetext{
12 Rojas Aravena, Francisco (Editor) Balance estratégico y medidas de confianza mutua. Santiago, Paz y Seguridad en las Américas/FLACSO-Chile/The Woodrow Wilson Center, 1996.337 pp.

Rojas Aravena, Francisco (Editor) Medidas de confianza mutua y verificación. Santiago. Paz y Seguridad en las Américas/FLACSO-Chile/The Woodrow Wilson Center, 1996.272 pp.
} 
Si desarrollamos MCM, como parte de un proceso de creación de un clima de confianza, podremos interactuar de manera más transparente y predictible. Posibilitarán un mayor espacio para la diplomacia y la política. Las medidas de confianza mutua generan garantías. Allí radica la diferencia entre fe y confianza. Con base en las garantías podremos establecer un patrón objetivo de evaluación, independientemente de nuestra fe en el grado de cumplimiento con el que iniciamos el proceso.

Las medidas de confianza mutua son acciones bilaterales y multilaterales destinadas a prevenir situaciones de crisis y de conflicto. Contribuyen a la comunicación entre los actores. Crean una atmósfera propicia para establecer un marco de entendimiento que objetivice las percepciones de amenaza inmediata y eviten riesgos y elementos de sorpresa. Las MCM presuponen la existencia de diferencias de intereses y baja confianza en las relaciones; pero a la vez, una voluntad política básica tendiente a evitar el enfrentamiento y abrir opciones para la solución del problema. Su aplicación es fundamental cuando las diferencias pudiesen expresarse por medio del uso de instrumentos de fuerza. En esa situación un error de interpretación desencadena un conflicto no deseado.

Las medidas de confianza mutua son acciones con una vinculación de reciprocidad necesaria, no necesariamente equivalente, pero sí paralela en el tiempo. Una rápida secuencia temporal producirá un efecto de progresividad y verificación simultáneamente. Un paso es seguido por otro, siempre y cuando la contra parte esté cumpliendo con el compromiso recíproco. Diez características contribuyen a perfilar de manera más clara a este instrumento de la seguridad internacional: 1) transparencia y apertura; 2) predictibilidad; 3) reciprocidad y equivalencia; 4) mejoramiento de la comunicación; 5) estabilizar y proyectar las relaciones; 6) factibilidad y realismo en la ejecución; 7) coherencia con otras políticas; 8) verificables; 9) contar con soporte social; y, 10) diferenciación bilateral, subregional o multilateral.

Las MCM son un instrumento, una técnica, para el mantenimiento de la paz; no resuelven el conflicto o la diferencia de intereses; posibilitan la comunicación y con ello hacen más transparentes y predictibles los cursos de acción de los diversos actores involucrados. Cabe destacar que el proceso presupone buena fe y la voluntad de evitar el enfrentamiento. En caso contrario, las MCM pueden ser utilizadas para ganar tiempo o para intentar ocultar los verdaderos intereses de un actor sobre otros, y crear un falso sentido de 
seguridad. Las Medidas de Confianza Mutua buscan evitar la escalada como respuesta automática. Para ello es fundamental generar confianza, establecer redes de comunicación e interpretación, de decodificación de acciones y generar espacios para la verificación. Al establecer la circulación y entrega de información básica sobre los cursos de acción, y al refrendarse en hechos, es posible regular ciertas formas de comportamiento. Así se evitan interpretaciones equívocas y a partir de ellas se gatille un conflicto. La confianza crea confianza. La confianza potencia la cooperación.

Si hay compromisos sólidos en lo diplomático, un clima general de confianza y cooperación, se estructurará un sistema de MCM verificables en el sector defensa, se generarán espacios para establecer medidas de control y limitación de armamentos, en áreas definidas concertadamente. Las MCM no son medidas de control de armamentos. Tampoco son medidas de limitación de armamentos, ni constituyen medidas de desarme. Aunque el desarrollo de las MCM pueda formar parte de un proceso que incluya medidas de esas características. Es difícil pensar que puedan desarrollarse medidas de control y limitación de armamentos y más aún de desarme, en ausencia de un contexto de confianza, en donde se focalizan y usan las MCM.13

\section{¿Cómo evaluar las MCMyS?}

Sin lugar a dudas una de las áreas que ha experimentado un salto cualitativo en la cooperación es el referido a las medidas de confianza mutua y de seguridad (MCMyS). Los países de la región, en un corto período de tiempo, concordaron una serie de medidas que han puesto en ejecución, sin dilación, en relación con la creación de confianza a nivel militar; en especial en lo referido a cuestiones estratégicas. Si uno mira la situación hace una década, las medidas de confianza mutua eran reducidas y débiles, con un carácter esencialmente protocolar entre institutos armados. Al finalizar el siglo las medidas de confianza mutua constituyen un elemento crucial en la construcción de parámetros de cooperación e integración en cuestiones de defensa en las

${ }^{13}$ En el hemisferio la experiencia así lo muestra. Luego de avanzar en las MCMyS se formalizó y abrió a la firma, el 7 de junio de 1999. en la Asamblea General de la OEA, la Convención Interamericana sobre Transparencia en Adquisiciones de Armas Convencionales. 
diversas subregiones. Lo anterior significa fundamentalmente que se ha estructurado un conjunto de mecanismos de prevención, que facilitan la cooperación. Ello no debe ser confundido con un reemplazo de las concepciones nacionales sobre seguridad o que estos diseños presuponen la eliminación de la conflictividad. Por el contrario, el conflicto entre Ecuador y Perú mostró de manera simultánea la vigencia de la conflictividad y como ésta puede escalar y, por otro lado, cómo las medidas de confianza y de seguridad pueden contribuir a desescalar la tensión militar como producto de evitar errores de interpretación que pudiesen conllevar decisiones erradas con un alto costo. La resolución de la conflictividad continúa siendo una responsabilidad política, de las más altas autoridades de los países y de los líderes del hemisferio. ${ }^{14}$

Las MCMyS buscan transferir estabilidad sobre la base de la predictilibilidad. Corresponden a un proceso que avanza en forma progresiva desde la prevención de riesgos hacia un proceso de cooperación que incrementa la interdependencia. Si bien ningún proceso puede señalarse como irreversible, la creación de un marco de relacionamiento de cooperación ampliado y de interdependencia incremental en los ámbitos político, económico, cultural, social y académico, estructurará un red de relaciones de una densidad tal que evitará que un conflicto particular adquiera una envergadura tal que ponga en cuestión el conjunto de la relación. De hecho, lo que hacen las medidas de confianza mutua es precisamente generar transparencia en el ámbito más sensible, el de la defensa para producir predictibilidad en lo referido al uso de la fuerza.

En el proceso de evaluar las medidas de confianza mutua y de seguridad podemos distinguir cuatro etapas diferenciadas:

La primera etapa corresponde a erradicar la desconfianza. Esta etapa busca actuar esencialmente sobre los elementos que amenazan a la relación y por lo tanto lo que buscan es evidenciar que no existe un comportamiento ofensivo o amenazante. En este primer nivel se destacan los siguientes tipos de medidas: ${ }^{15}$

\footnotetext{
4 En este sentido el mandato de los Presidentes y Jefes de Gobierno sobre la necesidad de abordar, en el marco de una Conferencia Especial de la OEA, el concepto de seguridad es de crucial importancia. Ello obligará a revisar el TIAR y otros mecanismos y regímenes internacionales en el hemisferio. Al respecto, ver resolución OEA. Asamblea General Resolución 1643, 7 de junio, 1999.

is En los informes nacionales presentados en la OEA, en las reuniones de Ministros de Defensa y en los informes de la IID, todos los países muestran medidas y acciones en este nivel. Clovis Brigagao y Marcelo Valle Fonrouge, "Argentina y Brasil: modelo regional de confianza mutua". En: Estudios Internacionales, No. 125. Santiagc, enero-abril, 1999. pp. 3-19. Patricio Rojas, "Seguridad regional: panorama y perspectivas". En: Estudios Internacionales, No. 119-120. Santiago, julio-diciembre, 1997. pp. 407-422.
} 
- Prevención del conflicto.

- Construir líneas de comunicación.

- Erradicación de las fuentes de sospecha.

- Mejorar en el conocimiento mutuo.

- Establecimiento de principios rectores de la "nueva" relación.

- Avances en acciones unilaterales.

La segunda etapa corresponde a la construcción de la confianza; es decir, establecer un conjunto sistemático de acciones que permitan estructurar un nuevo patrón de relación. Este se construirá a partir de generar una nueva oportunidad a la vinculación en un marco de reconocimiento de situaciones potencialmente amenazantes, pero que al haber avanzado de manera efectiva en el primer nivel, y en especial en las líneas de comunicación y transparencia básica, posibilita desarrollar acciones de cooperación de mayor efectividad. En esta etapa el diseño de marcos institucionales y la definición de arquitecturas de relacionamiento más permanente, cumplen un rol fundamental. Serán estas instancias de carácter regular donde se podrán abordar los problemas del relacionamiento y encontrar mecanismos para satisfacer los intereses de cada parte y formas para aminorar o abolir percepciones de amenaza referidas al uso de la fuerza. ${ }^{16}$ Lo anterior no significa que se deseche el modelo de disuasión como modelo estructural en la defensa nacional. Por el contrario, las medidas de confianza mutua pueden vincularse a una efectiva política de carácter disuasivo, pero que posee un eminente carácter defensivo en su concepción estratégica. Las principales acciones y medidas en esta etapa corresponden a las siguientes:

- Establecer un proceso con promesas satisfechas por medio de acciones efectivas.

- Establecer marcos de regulación institucionales, sobre la base de los principios establecidos y de la constitución de mecanismos operacionales específicos.

- Evidenciar y desarrollar una fuerte coherencia entre las medidas domésticas, los acuerdos internacionales y el "nuevo" patrón de relación que se busca afianzar.

\footnotetext{
${ }^{16}$ En la ültima década se han creado una serie de mecanismos bilaterales de diấlogo en cuestiones de seguridad. Cabe destacar el Comité Colombo-Venezolano, el Comitẽ Permanente de Seguridad ChilenoArgentino, el Memorándum de Entendimiento entre Argentina y Brasil. También diversos países han establecido mecanismos regulares de diálogo bilaterales con Estados Unidos. Otros han generado acuerdos subregionales como el Regional Security System en el Caribe y el Tratado de Seguridad Democrática de Centroamérica.
} 
La tercera etapa corresponde a la profindización de la confianza. En esta etapa se ha producido un incremento en las diversas áreas de interrelación. Las vinculaciones económicas, comerciales y financieras se ejecutan con una alta normalidad entre un significativo número de actores. Los conflictos y diferencias que surgen en este terreno no desbordan hacia otras áreas, en particular la política. El uso de la fuerza no aparece como opción frente a ningún conflicto en el área económica, la cual posee sus propios mecanismos de resolución de controversias. En esta tercera etapa la generación de acciones asociativas y cooperativas alcanza un nivel importante. Estas acciones se manifiestan en el terreno de la defensa y las cuestiones referidas al uso de la fuerza, pero van más allá de ellos. La cooperación aparece de manera transversal en el conjunto de la relación, de allí que los desarrollos en cada área particular refuerzan la perspectiva global. De igual forma los conflictos sectoriales aunque pueden adquirir niveles significativos, encuentran en este marco de cooperación incentivos prácticos y una proyección estratégica que busca su resolución al más corto plazo. En este nivel podemos destacar acciones en las siguientes áreas:

- Generación de acciones asociativas.

- Desarrollo de actividades conjuntas.

- Iniciativas con capacidad de amplificación.

- Avances hacia medidas duras, de alta sensibilidad.

Cuando se ha alcanzado el tercer nivel es posible avanzar hacia medidas efectivas tendientes a alejar aún más las potenciales percepciones de amenaza referidas al uso de la fuerza en el marco de la relación. ${ }^{17}$ Lo anterior significa que la potencialidad del uso de la fuerza se retarda y la cantidad de mecanismos de acción a ser usados, aún ante situaciones críticas, ofrece el más amplio abanico de oportunidades antes de que cualquiera de los actores considere la movilización del uso de la fuerza en una perspectiva disuasiva y defensiva como un elemento significativo. Más aún, las experiencias recientes en Europa evidencian que en el marco de los países asociados en la Comunidad, el uso de la fuerza no aparece bajo ninguna circunstancia en el horizonte,

\footnotetext{
"En el caso de las relaciones entre Argentina y Brasil, entre Chile y Argentina, podemos evidenciar que se ha avanzado de manera sustantiva en la construcción de la cooperación y la profundizaciōn de la confianza. Los tres gobiernos han publicado y hecho transparentes sus políticas de defensa (libros de la defensa), han programado y efectuado ejercicios militares conjuntos y buscan nuevas formas de cooperación. Por ejemplo, los ejercicios "Viekaren Il". El Mercurio, Santiago, 7 de septiembre, 1999.
} 
aunque sí puede estarlo de manera muy rápida fuera del ámbito geográfico cooperativo. Lo anterior presupone la efectivización de medidas que eliminan la percepción de amenaza al interior del espacio geográfico integrado. La ejecución plena de este nivel da paso a un cuarto nivel.

La cuarta etapa corresponde a la planificación de la cooperación. Al establecimiento de una alianza político estratégica sustantiva en la cual la conformación de estructuras, vínculos, redes de interdependencia, son lo característico. Es el momento del establecimiento de políticas comunes entre los distintos actores incluidas las políticas exteriores y de seguridad. La principal acción en este campo corresponde a:

- Estructuración de una red interdependiente.

En el ámbito específico de las MCMyS los avances se evalúan al considerar el exitoso y positivo grado de cumplimiento por parte de los diversos países de la región con lo establecido en las declaraciones de las conferencias regionales sobre la materia que surgieron en Santiago,1995 y EI Salvador,1998.

A modo de síntesis, podemos afirmar que se esta escribiendo una nueva historia de cooperación en materia de construcción de confianza. El grado de predictibilidad se ha incrementado. El desarrollo de acciones de primera generación, en especial de conocimiento, contactos, diálogo, se ha cumplido plenamente entre los países mayores de la región. En algunas subregiones se ha avanzado en el establecimiento de la confianza y, por lo tanto, se puede proyectar de manera positiva al futuro. Esto posee particular importancia en el Cono Sur, en Centroamérica y en el Caribe. Para ello se han diseñado mecanismos institucionales bilaterales y subregionales. No obstante, es necesario estructurar una mirada y un accionar más coherente en el plano hemisférico. La construcción de un régimen de seguridad coherente permitirá avanzar sobre los temas generales referentes a la seguridad y en la creación de regulaciones específicas en áreas determinadas o en regiones geográficas particulares. Los regímenes funcionales permiten profundizar acuerdos en áreas particulares o profundizar los entendimientos en subregiones específicas. La voluntad política es la que definirá el énfasis en cada caso. El diseño "arquitectónico", en todo caso, cumple un rol muy importante. El hemisferio debe decidir sobre el reemplazo del Tratado Interamericano de Asistencia Recíproca (TIAR), ya sea por la vía de una modificación profunda que le genere una nueva legitimidad o bien reemplazarlo por un nuevo Tratado.

Lo anterior es fundamental. Más aún, si consideramos las afirmaciones 
centrales del Secretario General de la OEA, César Gaviria ${ }^{18}$, señaladas en la Conferencia Regional de la OEA sobre MCMyS, efectuada en Santiago en 1995, las que siguen teniendo plena vigencia:

1.- El hemisferio no posee una agenda de seguridad lo suficientemente refinada y capaz de reemplazar a las orientaciones que se impusieron durante la guerra fría.

2.- Aún se esta lejos de haber llegado a un consenso sobre los objetivos, políticas e instrumentos para la cooperación multilateral.

3.- No se puede asumir que la democratización es irreversible

4.- El diseño de una visión estratégica será tarea compleja.

5.- Existe una ventana de oportunidad para transformar las relaciones político-militares en el hemisferio, para reflejar intereses colectivos.

La emergencia de la nueva agenda de seguridad evidencian una alta heterogeneidad entre las distintas subregiones del hemisferio, lo que complejiza aún más el diagnóstico anterior. De allí la necesidad de avanzar hacia la conformación de un concepto común sobre seguridad, como lo establecieron los Presidentes en la II Cumbre de las Américas; cuestión sobre la cual la Comisión de Seguridad Hemisférica ha venido trabajando en los últimos meses. ${ }^{19}$

\section{Recomendaciones de política ${ }^{20}$}

Los cambios internacionales han generado incertidumbre en los países latinoamericanos. Esta se expresa tanto en la reconceptualización de la seguridad, en la redefinición de las amenazas, como en las misiones que deben cumplir las fuerzas armadas. El establecimiento de regímenes internacionales de cooperación posibilita establecer un marco de acción conjunta y colaborativa en el hemisferio. Las medidas de confianza mutua y de seguridad se ubican en este campo.

\footnotetext{
${ }^{18}$ César Gaviria, "Oiscurso I Conferencia Regional sobre Medidas de Confianza Mutua". En: Fuerzas Armadas y Sociedad, Vol. 10, No. 4, octubre-diciembre. FLACSO-Chile. Santiago, 1995.

19 La Comisión de Seguridad Hemisférica durante la Presidencia del Embajador Carlos Portales, organizó -con el apoyo del Gobierno de Chile- un foro académico sobre la seguridad hemisférica, en donde se analizaron las cuestiones centrales que dicen relación con la formulación de un concepto de seguridad adecuado a los desafios de la posguerra fria y que de cuenta de los avances en la cooperación subregional $y$ hemisférica.

zo Rojas Aravena, Francisco (Eđitor) Cooperación y seguridad internacional en las Américas. Editorial Nueva Sociedad (1999). En prensa.
} 
La construcción de un sistema de regímenes internacionales de carácter vinculantes que posibiliten conformar áreas de estabilidad y seguridad internacional en cada una de las subregiones y de manera agregada en el hemisferio, es esencial. En este camino aparecen una serie de recomendaciones de políticas que apuntan hacia el desarrollo de la cooperación para la paz y una perspectiva más general hacia la desmilitarización de las relaciones interestatales.

1.-Reafirmación y ratificación de los compromisos hemisféricos y regionales de solución pacífica de las controversias.

2.- Cooperación para desactivar conflictos fronterizos. En este ámbito se ubican las acciones referidas a una activa política diplomática, que vincule tanto los aspectos militares como las relaciones económicas, comerciales y culturales. El desarrollo de medidas de confianza mutua se ubican preferentemente en este nivel. De ellas podemos destacar: el establecimiento de comisiones de fronteras, intercambio de información y de visitas, mejorar los sistemas de comunicación, notificación previa de actividades militares en zonas fronterizas, etc.

3.- Cooperación preventiva. La cooperación para prevenir acciones de terrorismo transnacional o de acciones de mafias internacionales en zonas de frontera puede ser un elemento clave para evitar conflictos. En este ámbito pueden desarrollarse medidas como el patrullaje conjunto de fronteras, y el intercambio de informaciones.

4.- Cooperación técnica. La cooperación científica y tecnológica es una esfera muy amplia, puede abarcar desde proyectos de desarrollo industrial tecnológico conjunto como por ejemplo, poner un satélite de comunicaciones de uso colectivo, hasta medidas puntuales de carácter técnico que posibilite la verificación de otros acuerdos. También en este ámbito puede caber la investigación científica vinculada a temas de carácter subregional o regional, como por ejemplo, el deterioro de la capa de ozono o el cumplimiento de los tratados antárticos.

5.- Cooperación para el desarrollo. El surgimiento de nuevas amenazas, por ejemplo, las vinculadas a la seguridad ecológica requiere de actitudes cooperativas. Los procesos de integración económica y el uso de recursos compartidos, fomenta la cooperación en este ámbito.

6.- Cooperación para la seguridad regional, hemisférica y global. Cada una de las acciones desarrolladas en los ámbitos de la confianza, del control, la limitación y la reducción reafirman las tendencias a construir una zona más 
estable. Pero además la región puede realizar un aporte importante en misiones de observación de la paz, verificación y establecimiento de la paz, ejecutadas bajo el mandato de la ONU o de la OEA.

7.- Monitoreo permanente de las actividades desarrolladas en las mismas subregiones. 